\title{
Pensamiento Crítico en egresados de Comunicación Social y Periodismo de la Universidad Surcolombiana
}

Fernando Charry González *

Rodolfo Llinás, el neurocientífico colombiano que ejerce como director del Departamento de Fisiología y Neurociencia en la Universidad de Nueva York, insiste en que "a la gente hay que enseñarle a pensar". Llinás defiende ante todo la preeminencia del pensamiento, la exalta y aboga por ella en sus múltiples entrevistas, como en la de El Tiempo el 22 de febrero del 2011 en la que habla acerca del vínculo entre la educación y la producción científica del país. Dice: "Hay que tener no solo conocimiento y equipos; también gente a la que se le deje inventar, a la que se le deje pensar [...] Hay que enseñarle a la gente a pensar y a que invente sus propias soluciones".

A este planteamiento le añadiría que hay que enseñarle a la gente a pensar críticamente. Es decir, que su pensamiento sea crítico. Tal afirmación no la reduzco al acto simple de ir en contra vía de los argumentos o de las propuestas del otro -lo que algunos llamarían pensamiento divergente- sino más bien de valorar desde todos los ángulos posibles una situación -no necesariamente problemática- y proponer una lectura que dé luces hacia la construcción de soluciones pertinentes. Allí radica, considero yo, uno de los grandes beneficios al momento de propiciar el pensamiento crítico en la educación: con ella se puede valorar la realidad siempre y cuando la pensemos en términos dictados por la razón.

Sobre la necesidad de pensar correctamente se ha dicho bastante, desde la antigua Grecia hasta nuestros días. No obstante, a mediados del siglo pasado surgió el estudio multidisciplinario del pensamiento que abordó el tema desde la sociología, la sicología, la pedagogía, entre otras muchas áreas del conocimiento. Todas ellas han acertado a lo que se conoce como Pensamiento Crítico. Aportes como los de Dressel y Mayhew en 1954, o los de Jhon E. McPeck en 1981, pasando por los del Nickerson y Richard Paul en los últimos años del siglo pasado hasta llegar al concepto de Robert Ennis, en quien reconozco los aportes más significativos en el debate de lo que se puede entender como Pensamiento Crítico, ya que él manifiesta que dicho pensamiento es aquel "reflexivo y razonable que se centra en que la persona pueda decidir qué creer o hacer, buscar la verdad en las cosas y llegar a conclusiones razonables con criterios y evidencias. Resolver problemas y tomar decisiones" (Ennis, 10).

A la luz de lo anterior vale la pena preguntarse por el papel de la educación y la necesidad de fomentar el pensamiento crítico en los estudiantes de todos los niveles educativos. Dicha pregunta surge en mi condición de docente de un programa de Comunicación Social y Periodismo de Universidad Pública. Así pues, en el marco de la Maestría en Educación que adelanté en la Universidad Surcolombiana, me di a la tarea de cotejar las características que esgrimen los autores del pensamiento crítico con lo expresado en el proyecto formativo del programa del cual hago parte, tomando como referente la misión, los aspectos teleológicos y el perfil profesional del mismo con el ánimo de establecer si la formación de pensamiento crítico de nuestros profesionales responde -si bien en los documentos del programa no está expresado directamente como propósito- a un propósito claro y estudiado o puede resultar de un ejercicio retórico para enriquecer la redacción del documento y de la propuesta contenida.

* Magíster en Educación Universidad Sulcolombiana 
Tratar de responder a la anterior pregunta ameritaría un estudio más exhaustivo y prolongado que tomaría años de juiciosa investigación académica. No obstante, considero relevante el estudio del pensamiento crítico a una escala más modesta -aunque no menos importante-; en ese sentido tengo en cuenta la diversidad de desempeños laborales y profesionales de egresados nuestros en el campo de la Educación, Medios de Comunicación tradicionales al igual que Comunitarios y Ciudadanos, liderando proyectos comunicativos en comunidades y organizaciones, entre otros.

De ahí que me haya aventurado por estudiar las experiencias significativas reconocidas por los egresados del programa de Comunicación Social y Periodismo, de la Universidad Surcolombiana como decisivas en la formación de su pensamiento crítico para hacer una evaluación cualitativa de los procesos curriculares y extracurriculares de los egresados del programa de Comunicación Social y Periodismo, de la Universidad Surcolombiana.

Para dicho fin me serví del método de los relatos de vida, puesto que éste me permitió sondear en la subjetividad humana a través del lenguaje (Packer, 2013), todo con el propósito de recuperar la importancia de la oralidad como fuente de información en la investigación cualitativa. Entendemos el relato de vida como el relato que aspira a producir narraciones de los actores sociales colaboradores, en torno a experiencias particulares asociadas con el tema de la investigación (Medina, 2008).

Para ello llevé a cabo la selección de los participantes de la investigación, los cuales obedecieron a criterios cronológicos (año de graduación) y su activa participación a lo largo del proceso de consolidación del programa. Con dicha selección busqué explorar la subjetividad del egresado para hacer emerger esas características propias de quien ha fomentado en su formación el Pensamiento Crítico. De allí emergieron, ya en el proceso de análisis, dos líneas gruesas de acción: procesos curriculares y procesos extracurriculares, de los cuales, a su vez, se desprendieron algunas subcategorías así: En los procesos curriculares; Profesores, Asignaturas y Otras Experiencias Curriculares. $\mathrm{Y}$ en procesos extracurriculares; intramuros y extramuros.

Seguido adelanté una triangulación analítica entre los hallazgos más relevantes de dichas respuestas, la teoría que sustenta desde una perspectiva académica los resultados y mi aporte como investigador y docente. Este ejercicio me permitió concluir que los procesos significativos reconocidos por egresados del Programa de Comunicación Social y Periodismo de la Universidad Surcolombiana para su desempeño profesional y laboral, de conformidad con el perfil definido para ellos y la formación de un pensamiento crítico, reflejan hasta qué punto influyeron en ellos el plan de estudios, los docentes y el contexto universitario. Todos estos elementos constituyen el caldo de cultivo de sus experiencias vitales y académicas, consolidaron sus ideas, manifestaron su posición crítica frente a diversos temas, actuaron de conformidad a lo que se esperaba de ellos desde la academia.

Según Lipman (2001) la autonomía y el pensamiento de orden superior son manifestaciones inseparables para definir el pensamiento crítico en los seres humanos. Afirma el autor que la autonomía no debe confundirse con un acentuado sentido de la individualidad (individualismo) sino con realizar sus propios juicios sobre los sucesos, fomentar su propia comprensión del mundo y construir sus propias concepciones sobre la clase de personas que quieren ser y el tipo de mundo en el que quieren vivir. De igual modo, Lipman manifiesta que el pensamiento de orden superior es un pensamiento rico conceptualmente, coherentemente, organizado y persistentemente exploratorio. Estas dos 
cualidades fueron encontradas en algún grado (mayor o menor) en los participantes del estudio. De acuerdo a lo anterior y a lo consignado en los relatos de vida se puede manifestar que el egresado de nuestro programa posee, alcanza o logra dos de las características referidas por los teóricos del pensamiento crítico como se demuestra en mayor medida en la sección de Procesos Extracurriculares y en los demás secciones, en menor medida.

Así mismo, de conformidad con lo expresado por Ennis, los testimonios de los egresados dan cuenta de un pensamiento que evidencia reflexividad, que se presenta como razonable y en el que, además, se denota la libertad que tienen éstos para decidir, a partir de juicios construidos analíticamente, qué creer o hacer. Lo anterior se ve reflejado en los momentos en los cuales los egresados dicen manifestar en sus testimonios la capacidad de analizar resultados y situaciones del contexto en el cual se desempeñan laboralmente; pero, también, cuando dicen hacerlo con el propio sujeto. Y se entiende que sus pensamientos sean razonables puesto que se evidenció asimismo una capacidad de analizar situaciones, información, argumentos que buscan la verdad en las cosas para llegar a conclusiones razonables con criterios y evidencias.

De este modo podemos colegir que los egresados entrevistados se apropiaron de herramientas para identificar problemas y diseñar propuestas y estrategias para resolver los mismos, no solo a nivel comunicativo, sino también de otras dimensiones; es decir, dieron cuenta de un pensamiento autónomo y de orden superior. Pero lo que llama más la atención de todos ellos es que dicha capacidad de resolver problemas no se limita únicamente al plano teórico sino que, una vez tomada la posición crítica, son abocados a llevar a cabo acciones frente a dichos problemas. Son capaces, en el decir de Stenberg (citado por Lipman) de generar procesos, estrategias y representaciones mentales que la gente utiliza para resolver problemas, tomar decisiones y aprender nuevos conceptos.

Ahora bien, dicha capacidad de realizar acciones encaminadas a resolver problemas, es producto de una orientación para asumir actitudes críticas frente a la realidad del entorno, lo que ayudaría a la conformación de un pensamiento crítico durante los años universitarios. Esto queda claro en los testimonios, en la opiniones particulares que el grupo de entrevistados tienen sobre los profesores y sobre las asignaturas. De ahí que consideremos que el Programa de Comunicación Social y Periodismo de la Universidad Surcolombiana aporta a dicha construcción de pensamiento crítico por cuanto se ha empeñado -así lo reconocen también los entrevistados- en diseñar un currículo que al menos contempla procesos que propenden por estimular el pensamiento crítico en sus estudiantes.

Quizá uno de los ejemplos más inmediatos y elocuentes que posibilitan la consolidación de un pensamiento crítico sea el Programa de Comunicación Social y Periodismo de la Universidad Surcolombiana mismo. Allí, el cambio generacional ha permitido que egresados del programa se hayan vinculado como docentes para brindar lo que alguna vez les brindaron sus maestros: la posibilidad de establecer desde un punto de vista crítico un mapa de la región en la que están inmersos para ofrecer posibles soluciones que contribuyan a la solución de los problemas comunicativos.

De no haberse fomentado en ellos los principios necesarios para que desarrollaran actitudes críticas frente a su desempeño profesional y laboral en su mirada a las realidades de su entorno, habría sido muy difícil que pudieran aportar principios que fomentaran a su vez dicho pensamiento crítico. Pero ¿cuáles son esas particularidades del proceso vivido por los egresados del programa de Comunicación Social y Periodismo de la Uni- 
versidad Surcolombiana que les permitieron hacerse de características propias de las que he venido mencionando como parte del Pensamiento Crítico?

Considero que una de esas particularidades 0 , si se quiere, contingencias durante el proceso universitario vivido en el campus académico tiene que ver con la zona o espacio geográfico al cual pertenece la Universidad. Deleuze y Guattari (1993) manifiestan que la geografía no sólo es física y humana, sino mental, como el paisaje, y es la región que nos ha tocado en suerte un sistema geográfico de múltiples conflictos y procesos sociales, y eso, sin duda alguna, determina la construcción social y mental de sus habitantes. Dicha contingencia no es una excepción a la hora de abordar el ejercicio académico desde una institución de carácter público que propende hacia la construcción social.

Otro elemento que amerita una consideración especial y que por lo general no se toma en cuenta, es la naturaleza académica de los docentes. No hay nada oculto al decir que las Universidades Públicas Colombianas llevan a cabo su labor institucional de la mano de fuertes principios que las lleva a comprometerse con la realidad social y política de su región. Con esto no se quiere decir que la educación superior privada no tenga el mismo compromiso, simplemente que éstas, en consonancia con lo dicho por Chomsky, obedecen más a principios de mercado que a principios sociales generadores de cambio sociales. La gran mayoría de los docentes del Programa de Comunicación Social y Periodismo de la Universidad Surcolombiana provienen del primer tipo de Universidad señalada, lo cual permite que sus conocimientos, reflexiones y orientaciones intelectuales sean adquiridos a su vez por los estudiantes, todo lo cual deja ver un marcado interés por pensar la región de una forma crítica y constructiva, que permita la movilización social hacia caminos más equitativos y justos.

Añadido a lo anterior, se debe considerar que, como lo plantea Steiner (2004), dentro de su infinita complejidad y sutiles interacciones de transmisión de saberes, confianza y entusiasmo que el ejercicio docente puede generar, existen todos los matices imaginables en cuanto al trabajo de cada profesor, desde muestras claras de dificultades para enseñar, hastío y desencanto, hasta un elevado y misterioso don para educar y ser así reconocido como un maestro ejemplar, lo cual acaba aportando al modelo construido por los egresados en tanto que ven a muchos docentes (Iriarte, Torres, Cortés, etc.) como modelos intelectuales a seguir.

Por último, es interesante ver la analogía realizada por los egresados con respecto a que, para ellos, la Universidad - y el programa - son un mundo en pequeño que ayuda a las personas a vincularse a la realidad, a proponer, a tomar conciencia de los problemas que afronta la sociedad, lo cual se da no sólo desde el aula de clase sino desde el compromiso que algunos adquirieron desde distintas organizaciones.

Una vez realizada la respectiva evaluación cualitativa del proceso de formación de Pensamiento Crítico fomentado en los egresados del Programa de Comunicación Social y Periodismo de la Universidad Surcolombiana, se debe manifestar que, como lo plantea Martínez (2007), los resultado deben hacer parte de un proceso riguroso, sistemático y crítico: es decir, más científico. Que no sea el resultado de algo espontáneo, no programado sino de un componente académico estructural y pensado para ese fin; no obstante, desafortunadamente, ese no ha sido el caso del Programa, ya que en su currículo, principios teleológicos, etc., no se expresa de manera directa la intención de fomentar el pensamiento al que he venido aludiendo.

$\mathrm{Si}$ ben se infiere que existe un propósito por fomentar el pensamiento crítico consig- 
nado en la teleología del programa, este no se logra definir en acciones curriculares específicas; solo se notan algunos esfuerzos coyunturales o esporádicos en diferentes asignaturas. En cambio las experiencias extracurriculares y los desempeños laborales y profesionales han favorecido con mayor claridad el desarrollo de este tipo de pensamiento; un buen grupo de egresados nuestros son los que lideran la comunicación que hace resistencia a los proyectos minero energéticos que se construyen en la región (Represa el Quimbo), otros hacen participación política, algunos dedicados al fortalecimiento de medios y experiencias comunicativas comunitarias y ciudadanas, un grupo de comunicadoras lideran la campaña de no violencia contra las mujeres, otro tanto lidera el Centro de Análisis de la Información y la Comunicación -CAIC- que hace valoración a la información que los medios tradicionales producen en los temas neurálgicos de la región.

Existe, por el contrario, una cierta superficialidad ligada al ejercicio retórico en los documentos atrás mencionados (currículo, principios teleológicos, etc.), en tanto que en estos se manifiesta la intención de formar un profesional con características donde sobresalga la excelencia humana y académica que aporten profesionalmente a la región, pero no a contribuir al cambio de mentalidad profundo, que se sienta la emancipación y la autonomía mental necesaria para consolidar un verdadero pensamiento crítico.

Por lo anterior considero que el programa debe proponerse orientar un proceso formativo que conduzca a lo manifestado por Paul y Elder (2005) en donde se considera que el pensamiento crítico es una vía para analizar y evaluar los procedimientos mentales de los sujetos con el propósito de mejorarlo, y donde éste (el pensamiento crítico) sea la clave para desencadenar el lado más creativo del pensamiento (la verdadera mejora del pensamiento) que reestructurar los procesos mentales como resultado de un procedimiento analítico y evaluativo eficiente.

De lo anterior se puede decir que el pensamiento crítico es susceptible de ser fomentado siempre y cuando se conjuguen elementos que permitan al estudiante potenciar su capacidad de análisis (un ambiente académico propicio, un plan de estudio pensado para este propósito y una planta docente comprometida con la independencia académica propositiva), todo lo cual va en consonancia con la crítica hacia el cauce neoliberal que ha tomado la educación superior, según Noam Chomsky, pero a su vez a la integralidad que supone los postulados de Rodolfo Llinás, con lo cual el conocimiento y el pensamiento serán la virtud con la cual la educación colombiana renovará sus aires tanto en lo investigativo como en lo que corresponde a la innovación.

\section{Renovar currículos para fomentar el pensamiento crítico}

El programa de Comunicación Social y Periodismo de la Universidad Surcolombiana debe asumir la formación y fomento del pensamiento crítico. Para ello es necesario llevar a cabo un proceso de renovación curricular orientado a ese propósito, es una demanda académica y para el desarrollo del sujeto en tiempos en que pensar críticamente se ha vuelto una necesidad más que una opción.

El camino hacia el pensamiento crítico que se sugiere para el programa debe contemplar dos factores. El primero es el reconocimiento histórico de los procesos vividos al interior del programa, el cual debe contemplar no solo los distintos diseños curriculares que ha tenido, sino también las luchas por la consolidación de un programa que ha tenido como misión la de brindar profesionales que aporten a la construcción social; así mismo es necesario reconocer la importancia de los actores que han hecho parte de dicho proceso histórico (docentes, decanos, egresados, etc.), ya que esto permitiría 
la articulación entre el pasado, el presente y daría un derrotero para afrontar el futuro que se desea proyectar.

Por último quiero poner el acento en la importancia de los aportes que los egresados del programa hacen en esta investigación. Las renovaciones que se contemplen deberán reconocer las experiencias visibilizadas por los egresados y convertirlas en estrategias pedagógicas o curriculares que sean asumidas por el conjunto de los docentes para que permitan la consolidación del pensamiento crítico en el programa.

\section{REFERENCIAS BIBLIOGRÁFICAS}

Aguilera S., Zubizarreta, Y, Magdalena, E \& Castillo, J. (2005) Estrategia para fomentar el pensamiento crítico en estudiantes de Licenciatura en Enfermería. Instituto Superior de Ciencias Médicas de La Habana.

Alejos, A, (2005) ¿Qué es el pensamiento crítico? Recuperado de: http://avita1706. blogspot.com/

Alvira, F. (1991): Fases en el desarrollo de una evaluación.Metodología de la evaluación de programas.Centro de Investigaciones Sociológicas, Cuadernos metodológicos, Número. 2. 2132.

Brunner, J, J, (15 de julio 2009) Principios Formativos. Columna de opinión publicada en Artes y Letras del diario El Mercurio. http://mt.educarchile.cl/MT/jjbrunner/archives/2007/07/principios_form.html

Deleuze, G \& Guattari, F (1993). ¿Qué es la Filosofía? Barcelona: Editorial Anagrama

Dressel, P.L., \& Mayhew, L.B., (1954). General Education: Explorations in Evaluation. Washington DC. American Council on Education.

Ennis, R. H., (1991). Critical thinking: A streamlined conception. Teaching Philosophy.

Gardner, Howard (2005) Las cinco mentes del futuro. Barcelona: Editorial Paidós.

Habermas, J. (1987) Teoría de la acción comunicativa. Barcelona: Taurus.

Hawes B., G., (2003) Pensamiento crítico en la formación universitaria. Instituto de Investigación y Desarrollo Educacional, Universidad de Talca. México.

Kurfiss, JC (1988) Critical thinking: theory, research, practice, and possibilities. ASHE-ERIC High

Limiñana Gras, R.M., Corbalán Berna, J. y Sánchez López, M.P. (2010). Creatividad y estilos de personalidad: Aproximación a un perfil creativo en estudiantes universitarios. Anales de Psicología, 26(2), 273-278

Lipman, M. (2001). Pensamiento Complejo y Educación. Buenos Aires: Ediciones de la Torre. (1985). Philosophy for Children and Critical Thinking. National Forum LXV. 
Llinás, R. (2011, 22 de febrero). 'Hay que enseñarle a la gente a pensar': Rodolfo Llinás. Periódico el Tiempo. Recuperado de: http://www.eltiempo.com/archivo/documento/ MAM-4410378

Marciales, G. P. (2003) Pensamiento crítico: Diferencias en estudiantes universitarios en el tipo de creencias, estrategias e inferencias en la lectura crítica de textos. Universidad Complutense de Madrid.

Martínez, M (2007). Evaluación Cualitativa de Programas. Caracas: Editorial Trillas.

Martinic, S (1998). Efectividad para el cambio. Evaluación de proyectos sociales con niños en América Latina. Santiago. Fundación Internacional para la Juventud.

Medina, P. (2008). El análisis y el problema teórico de los relatos de vida. En: Secretaria de educación y cultura. Métodos cualitativos aplicados II. Antología. Chihuahua: Centro de investigación y docencia. Maestría en Educación.

Muñoz, Ana. \& Beltrán, J. (2001) Fomento del Pensamiento Crítico mediante la intervención en una unidad didáctica sobre la técnica de detección de información sesgada en los alumnos de Enseñanza Secundaria Obligatoria en Ciencias Sociales. Departamento de Psicología Evolutiva y de la Educación, Universidad Complutense de Madrid. http://www.psicologia-online.com/ciopa2001/actividades/54/index.html

Nickerson, R. S. (1986). Teaching thinking skills. American Psychologist. Lawrence Erlbawm Publisher.

Packer, M. (2013). La ciencia de la investigación cualitativa. Bogotá. Uniandes.

Paul, R \& Elder, L (2005) Estándares de Competencia para el Pensamiento Crítico. Barcelona: Fundación para el Pensamiento Crítico.

Péréz, R. (2000). La evaluación de programas educativos: conceptos básicos, planteamientos generales y problemáticas. Revista de investigación educativa. Volumen 18. Número 2. 261-289.

Ruíz, C. (2001). Evaluación de Programas de formación de formadores. Recuperado en:http://www.tdx.cat/bitstream/handle/10803/5003/crb01de12.pdf?sequence=1

Steiner, G. (2004). Lecciones de los maestros. México: Fondo de Cultura Económica.

Tejada, C. Perfiles Profesionales. En: La biblioteca pública, nuevos espacios servicios emergentes. Editorial Trea

Torres, William Fernando (2000), Amarrar la burra de la cola. Bogotá: Libros del Olmo. Vattino, G., (1992) Más allá del sujeto. Barcelona: Ediciones Paidós. 